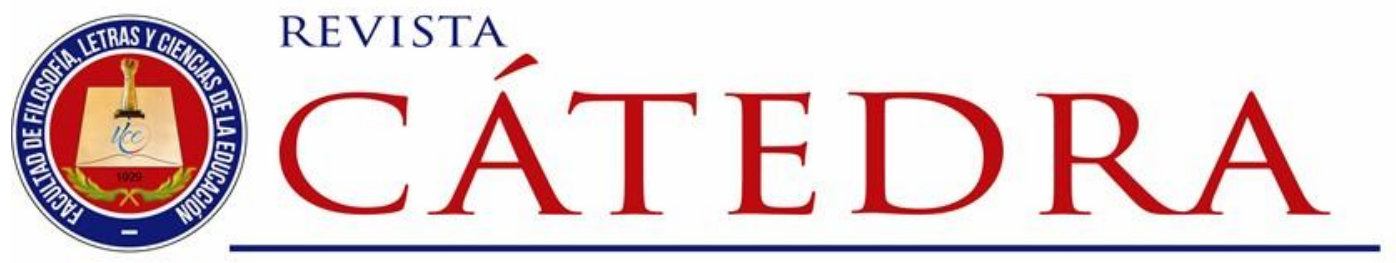

\title{
Aprendizaje significativo de la luminancia porel método punto por punto
}

\section{Significant learning of luminance by the point-by-point method}

\author{
José Ricardo Aulestia-Ortiz \\ Universidad Central del Ecuador \\ jraulestia@uce.edu.ec \\ https://orcid.org/0000-0001-5825-2487 \\ Shirley Vera-Macías \\ Universidad Central del Ecuador \\ ssvera@uce.edu.ec \\ https://orcid.org/0000-0001-7474-1483 \\ Nelson Mejía-Torres \\ Universidad Central del Ecuador \\ nbmejia@uce.edu.ec \\ https://orcid.org/0000-0001-9363-9505
}

Luis Puga-Peña

Universidad UTE

luis.puga@ute.edu.ec

https://orcid.org/0000-0003-1413-8070

(Recibido: 6/07/2019; Aceptado: 9/09/2019; Versión final recibida: 12/09/2019)

Cita del artículo: Aulestia-Ortiz, J., Vera-Macias, S., Mejía-Torres, N. y Puga-Peña, L. (2019). Aprendizaje significativo de la luminancia por el método punto por punto. Revista Cátedra, 2(3), 69- 82.

\section{Resumen}

Este artículo describe el proceso experimental realizado en la obtención de la luminancia de dos tipos de focos, el de incandescencia y el fluorescente en un mismo ambiente físico. Para realizar el mencionado estudio se eligió el método punto a punto, el cual, permite conocer la luminancia en puntos concretos de una superficie bajo una fuente de luz que se ubica a una determinada altura. Además, se trata de conocer el grado de confort visual del sentido de la vista en un lugar de iluminación uniforme. Los resultados obtenidos permiten realizar diversas comparaciones en el uso de una determinada lámpara, tomando en cuenta

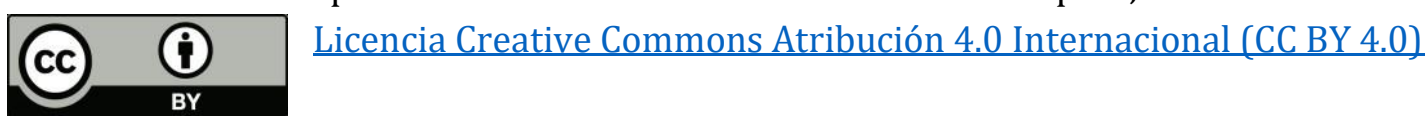

Revista Cátedra, 2(3), pp. 69-82, septiembre-diciembre 2019. e-ISSN: 2631-2875 
la que más beneficios presenta en función de ofrecer un nivel de luminancia y un confort visual apropiado, un ahorro económico y una fácil obtención y reemplazo.

La experiencia en el aprendizaje de Luz e Iluminación en busca de un aprendizaje significativo evoca conocimientos previos tales como: flujo, intensidad, ángulo sólido y luminancia con el objetivo de entender el fenómeno, presentar una nueva información y hacer un feedback en busca de una nueva información que acreciente su pensamiento en la vida cotidiana. Con los resultados obtenidos en la presente investigación se desea contribuya al mantenimiento de las luminarias de las aulas de la Carrera de Pedagogía de las Ciencias Experimentales Matemática y Física de la Universidad Central del Ecuador, las mismas que tienen características físicas similares al lugar en el cual se realizó la experiencia.

\section{Palabras clave}

Ángulo sólido, aprendizaje significativo, flujo, iluminación, luminancia, luz, medición.

\section{Abstract}

This article describes the experimental process performed in obtaining the luminance of two types of light bulbs, the incandescent and the fluorescent, in the same physical environment. To perform the mentioned study, the point-to-point method is chosen, which allows knowing the luminance at specific points of a surface under a light source located at a certain height; in addition, it is about knowing the degree of visual comfort of the sense of sight in a place of uniform illumination. The results obtained allow comparisons to be made in the use of a specific lamp, taking into account the one that presents the most benefits based on offering a level of luminance and appropriate visual comfort, economic savings and easy obtaining and replacement.

The experience analyzed allowed the application of the basic principles of light and lighting, subjects studied in the classroom, achieving a significant learning about: flow, intensity, solid angle and luminance, without neglecting the search for a pleasant atmosphere of a room through the qualitative and quantitative analysis of the phenomenon. In addition, it is expected that the results obtained in this research serve as a reference to initiate an improvement plan that contributes to the maintenance of the luminaries of the classrooms of the Pedagogy career of the Mathematical and Physical Experimental Sciences of the Central University of Ecuador, which have similar physical characteristics to the place where the research was carried out.

\section{Keywords}

Solid angle, meaningful learning, flow, lighting, luminance, light, measurement.

\section{Introducción}

El rol de la educación según la Ley Orgánica de Educación Superior (LOES) (Art 13. Literal

b) está el "promover la creación, desarrollo, transmisión y difusión de la ciencia, la técnica, la tecnología y la cultura" (p. 11). Este hecho inspira al presente artículo denominado aprendizaje significativo de la luminancia por el método punto por punto que tiene como objetivo principal el propiciar un espacio de estudio experimental para comparar la luminancia entre lámparas de diferente especie, ubicadas en un mismo entorno físico. Además, busca visibilizar el desarrollo y la obtención de un aprendizaje significativo de los principios de la luz e iluminancia con sus diferentes temáticas. 
Se plantea la comparación entre los resultados de la luminancia de una lámpara (foco) de luz de incandescencia y otro de luz fosforescente, situación que permitirá tomar una decisión adecuada en la elección de las lámparas que tiene más pertinencia en el uso diario, especialmente en los salones de clase que se desarrolla el proceso de enseñanza aprendizaje. Al respecto, Ausubel (1963), manifiestan que:

Un aprendizaje significativo se da cuando los contenidos son relacionados de modo no arbitrario y sustancial (no al pie de la letra) con lo que el alumno ya sabe. Por relación sustancial y no arbitraria se debe entender que las ideas se relacionan con algún aspecto existente específicamente relevante de la estructura cognoscitiva del alumno, como una imagen, un símbolo ya significativo, un concepto o una proposición (pág. 18).

Para lograr un aprendizaje significativo se debe aplicar metodologías activas como la experimentación, dando lugar a que los estudiantes apliquen los conocimientos anteriores en la consecución de un nuevo. De igual manera Ausubel, (1976), (2002); Moreira, (1997) citado por (Rodríguez Palmer, 2004) explican:

El aprendizaje significativo es el proceso según el cual se relaciona un
nuevo conocimiento o información con la estructura cognitiva del que
aprende de forma no arbitraria y sustantiva o no literal. Esa interacción
con la estructura cognitiva no se produce considerándola como un todo,
sino con aspectos relevantes presentes en la misma, que reciben el
nombre de subsumidores o ideas de anclaje (pág. 2).

Las ideas de anclaje o subsumidores adaptándolas al aprendizaje en el estudio de la iluminación artificial en las aulas constituyen un reto para conocer si el espacio físico en el cual se desarrollan las clases es agradable y de confort al ojo humano, ya que para realizar todas las actividades se requiere de una iluminación estable, esto plantea el buen uso de la iluminación artificial existente. El estudio observacional y de medición de la luminancia de una bombilla LED o de incandescencia y otra fluorescente ayuda a la comprensión y obtención de un aprendizaje significativo.

De acuerdo con Borja Reyes ( 2017) “Una buena iluminación es adaptable al lugar o local a iluminar creando un ambiente acogedor con un confort visual estable" (pág. 64). La permanencia de los seres humanos en una habitación depende mucho de la iluminación de los espacios que estén destinados para ocio, descanso o trabajo. La luz visible al ojo humano es una pequeña región del espectro electromagnético comprendido entre los $380 \mathrm{~nm}$ y $780 \mathrm{~nm}$ de longitud de onda, partiendo desde el ultravioleta al infrarrojo respectivamente. $\mathrm{Al}$ estudiar la naturaleza de la luz blanca se observa que el conjunto de longitudes de onda del espectro visible al atravesar un prisma cristalino el fenómeno de refracción mediante una gama de colores que va del infrarrojo al ultravioleta, verificándose que la longitud de onda en el infrarrojo es mayor que la del ultravioleta.

Un ambiente agradable produce comodidad al sentido de la vista, en el espectro visible se sabe que la luz amarillo-verde de longitud de onda $555 \mathrm{~nm}$ es la luz ideal, pero acompañada de una iluminación adecuada se puede desarrollar con mayor éxito cualquier actividad. Por ejemplo, iglesias, teatros, parques, calles, hospitales, aulas, museos, entre otras. Estos datos previos que el estudiante conoce y observa en la vida diaria sirven de cimiento en la puesta en práctica de su experimento y medición del fenómeno, siendo el estudiante el que desempeñe un papel activo, reestructurando y organizando la información. 
La Iluminación de superficies perpendiculares requieren ciertos niveles de flujo luminoso, siendo más intensa la iluminación en el punto central. Si se analiza un punto distante a la posición central la iluminación disminuye, por estar estrictamente relacionada con la intensidad luminosa y la altura, ya que la Iluminación es directamente proporcional a la intensidad luminosa e inversamente proporcional a la altura elevada al cuadrado.

\section{Materiales y métodos}

El método de investigación utilizado corresponde a un trabajo cuantitativo verificando la capacidad de dos bombillas de uso doméstico con el objetivo de lograr un aprendizaje significativo de la luminancia por el método "punto por punto". Para el efecto se ha recurrido a la experimentación en el laboratorio de la Unidad de Física de la Universidad Central del Ecuador. Además, se ha recopilado información bibliográfica de textos de física, simuladores y recursos tecnológicos de la Web. Todos los recursos mencionados se han identificado secuencialmente para poder hacer una experiencia de descripción cualitativa y cuantitativa de la aplicación de la luz e iluminación a través de un estudio descriptivo experimental completo de los fenómenos luminosos en el estudio del Movimiento Ondulatorio.

\section{Conceptos relacionados}

\subsection{Rayos de luz y sombras}

Las primeras propiedades de la luz estudiadas en el Movimiento Ondulatorio es la propagación rectilínea de la luz y sombras que se las comprende a través del sentido visual que ubica las distancias, direcciones y formas. Por ejemplo, el reloj solar de ingreso al teatro universitario de la Universidad Central del Ecuador produce la formación de una sombra nítida de un puntero de hierro que aprovecha la luz solar para medir el tiempo.

Considerando el principio de Huygens, cada punto en un frente de onda en movimiento puede considerarse como una fuente de ondas secundarias. El frente de onda en cualquier instante es la envoltura de estas ondas. Por tanto, según Young (2009) considera que "la luz emitida en todas direcciones por medio de la fuente puntual de luz puede representarse por una serie de frentes de onda esféricos que se mueven alejándose de la fuente a la rapidez de la luz" (pág. 1144).

Para nuestros propósitos, una fuente puntual de luz es aquella cuyas dimensiones son pequeñas en comparación con las distancias estudiadas. Al analizar el Principio de Huygens en las prácticas de laboratorio "Cuba de Ondas" se observó que los frentes de onda esféricos se vuelven prácticamente fuentes de onda planos en cualquier dirección específica a distancias muy alejadas de la fuente. Una línea recta imaginaria trazada perpendicularmente a los frentes de onda en la dirección de los frentes de onda en movimiento se llama rayo. Por supuesto, hay un número infinito de rayos que parten de la fuente puntual.

\subsection{Luz}

De acuerdo con León (2002) la "Luz es una manifestación de energía en forma de radiaciones electromagnéticas capaces de afectar el órgano visual, se denomina radiación a la transmisión de energía a través del espacio" (pág. 3), es decir, la luz se compone de partículas energizadas denominadas fotones, cuyo grado de energía y frecuencia determina la longitud de onda y el color.

La luz se la define como una radiación electromagnética que tiene un comportamiento isotrópico en todas direcciones sin necesidad de que exista un medio de propagación, La velocidad de propagación de la luz se establece en un valor de 299792458 m/s, aunque 
suele aproximarse a de $3 \times 10^{8} \mathrm{~m} / \mathrm{s}$. La velocidad de la luz está en perfecta armonía a la frecuencia y longitud de onda, siendo las unidades de la frecuencia los Hertz (Hz) y de la longitud de onda en nanómetros ( $\mathrm{nm}$ ). La luz visible es capaz de estimular el ojo humano a través de un espectro luminoso que va desde el ultravioleta al infrarrojo con valores entre los 380 a $780 \mathrm{~nm}$, respectivamente.

\subsection{Flujo luminoso}

Según Domínguez-Martínez (2008) el flujo luminoso "es la capacidad de radiación luminosa valorada por el ojo humano" (pág. 30). El flujo luminoso analizado como potencia radiante total emitida por una fuente de luz es capaz de afectar el sentido de la vista. Las fuentes de luz emiten energía electromagnética distribuida en múltiples longitudes de onda. La energía eléctrica suministrada a una lámpara emite radiación. Esta energía radiante emitida por la lámpara por unidad de tiempo se llama potencia radiante o flujo radiante. Sólo una pequeña porción de esta potencia radiante se encuentra en la región visible: la región está entre 380 (violeta) y $780 \mathrm{~nm}$ (rojo). Como lo predijeron las ecuaciones de Maxwell existen longitudes de onda por encima y por debajo de estos límites

El ojo humano no es sensible de igual manera a todos los colores. En otras palabras, iguales potencias radiantes de diferentes longitudes de onda no producen la misma brillantez. Una lámpara de luz verde de $40 \mathrm{~W}$ se ve más brillante que una lámpara de luz azul de $40 \mathrm{~W}$. La figura 1, indica la respuesta del ojo a diversas longitudes de onda. La curva de sensibilidad tiene forma de campana centrada aproximadamente en la región media del espectro visible. En condiciones normales, el ojo es más sensible a la luz verde-amarilla de longitud de onda de $555 \mathrm{~nm}$. Si las ondas electromagnéticas se organizan en un continuo de acuerdo a sus longitudes obtenemos el espectro electromagnético en donde las ondas más largas (longitudes desde metros a kilómetros) se encuentran en un extremo (Radio) y las más cortas en el otro (longitudes de onda de una billonésima de metros) (Gamma).

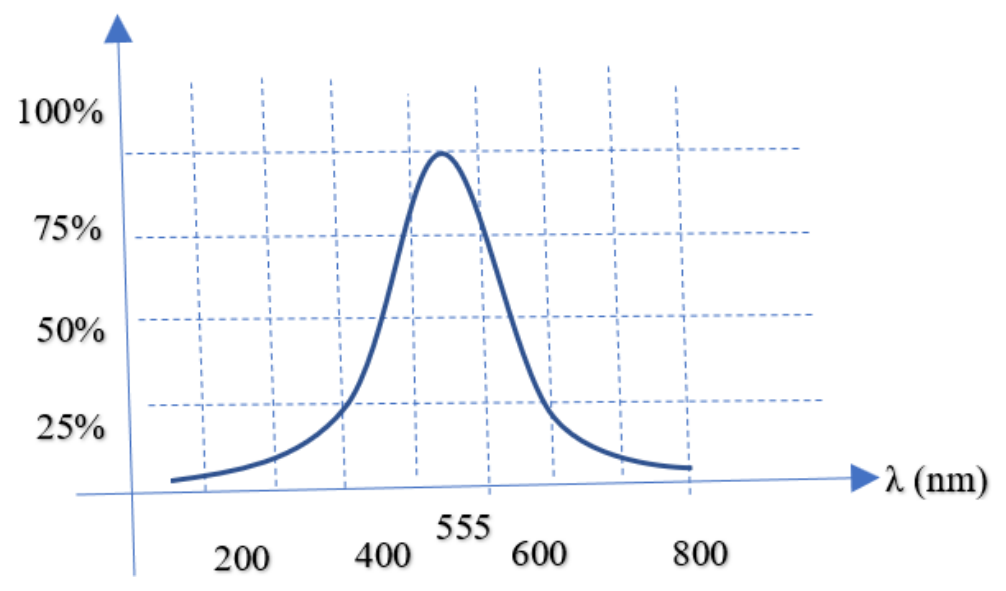

Figura 7. Sensibilidad de la luz al ojo humano

El flujo luminoso es la cantidad de energía en forma luminosa, emitida por una fuente. Su unidad es el lumen (Lm).

El concepto de ángulo sólido debe desarrollarse primero para referirse a un lumen. Un ángulo sólido en estereorradianes (sr), se define en la misma forma que un ángulo plano se define en radianes. 


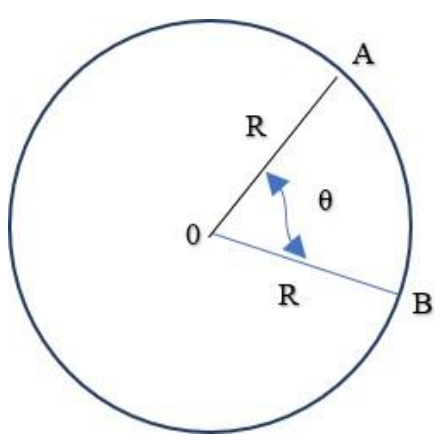

Figura 8. El radián

De acuerdo a la figura 2, cuando la longitud de arco $A B$ es igual al radio $\mathrm{R}$ se obtiene un radián. De la misma forma el ángulo sólido. Este puede pensarse como la abertura del extremo de un cono subtendido por un segmento de área sobre la superficie esférica.

Un estereorradián (sr) es el ángulo sólido subtendido en el centro de una esfera por un área sobre su superficie que es igual al cuadrado de su radio R. En general, el ángulo sólido en estereorradianes esta dado por:

$$
\Omega=\frac{\mathrm{A}}{\mathrm{R}^{2}}[\mathrm{sr}]
$$

Ecuación 1

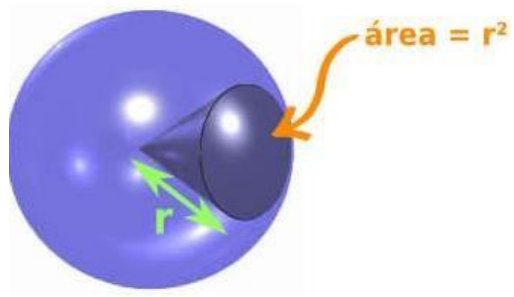

Figura 9. Estereorradián. Adaptado de (Disfruta de las matemáticas, 2011)

Así como en el ángulo plano $\theta$ para la obtención del radian, para la obtención del ángulo sólido de una esfera se hace la siguiente consideración:

$$
\Omega=\frac{4 \pi \mathrm{R}^{2}}{\mathrm{R}^{2}}
$$

Ecuación 2

Entonces se considera que: $\Omega=4$ л sr, que es independiente del radio de la misma, al definir un lumen desde el punto de vista de potencia afirma que "Un lumen (Lm) es el flujo luminoso o potencia radiante visible emitida desde una abertura de 1/60 de sección de $\mathrm{cm}^{2}$ de una fuente que emite luz formando espacialmente un ángulo sólido de 1 sr" (Tippens, 2007, pág. 652).

Una lámpara fluorescente puede emitir unos $4000 \mathrm{Lm}$, mientras que el flujo luminoso del Sol que ingresa por una ventana puede oscilar entre 2000 y $20000 \mathrm{Lm}$. Esta magnitud es característica de cada bombilla y, por tanto, es un dato facilitado por los fabricantes. La emisión de luz de los sólidos a temperaturas altas se establece una fuente patrón a la temperatura de solidificación del platino, aproximadamente $1773^{\circ} \mathrm{C}$. En la vida cotidiana se usa lámparas incandescentes que han sido calibradas por comparación con el patrón establecido para entender el fenómeno. 
La definición de la unidad de flujo luminoso afirma que "Un lumen es equivalente a 1/680 W de luz verde-amarilla de $555 \mathrm{~nm}$ de longitud de onda" (Tippens, 2007, pág. 652).

\subsection{Intensidad luminosa}

"Es el flujo luminoso por unidad de ángulo sólido en una dirección concreta. Su símbolo es I y la unidad en el sistema internacional es la candela (Cd)" ((INSHT) \& Alvarez Bayona, 2015, pág. 6). La intensidad luminosa (I) de una fuente de luz es el flujo luminoso (F) emitido por unidad de ángulo sólido $(\Omega)$ es:

$$
I=\frac{F}{\bar{\Omega}}
$$

Ecuación 3

La magnitud esencial de la intensidad luminosa del Sistema Internacional (SI) es la candela "cd".

$$
1[c d]=\frac{1[\mathrm{~lm}]}{1[\mathrm{sr}]}
$$

Ecuación 4

Por lo expuesto anteriormente el flujo luminoso es:

$$
\begin{aligned}
& \mathrm{F}=\mathrm{I} \cdot \Omega \\
& \text { Ecuación } 5
\end{aligned}
$$

y el flujo total de una fuente isotrópica es:

$$
\begin{aligned}
& \mathrm{F}=\mathrm{I} \cdot 4 \pi \\
& \text { Ecuación } 6
\end{aligned}
$$

\subsection{Iluminación o luminancia}

La iluminación o luminancia $(\vec{E})$ de una superficie $(A)$ se define como el flujo luminoso $(F)$ por unidad de área:

$$
E=\frac{E}{\mathrm{~A}}
$$

Ecuación 7

Sustituyendo el flujo luminoso y el ángulo sólido en la expresión anterior:

$$
\begin{gathered}
\vec{E}=\frac{\mathrm{I} \cdot \Omega}{\mathrm{A}} \\
\vec{E}=\frac{\mathrm{I} \cdot \frac{\mathrm{A}}{\mathrm{R}^{2}}}{\mathrm{~A}} \\
\vec{E}=\frac{\mathrm{I}}{\mathrm{R}^{2}} \\
\text { Ecuación } 8
\end{gathered}
$$

La unidad de iluminación E sus unidades es el Lux.

$$
1[l u x]=\frac{1[c d]}{1\left[m^{2}\right]}
$$

Ecuación 9 
La "luminancia" La ley de la inversa del cuadrado consiste en que el nivel de iluminación es proporcional a la intensidad luminosa e inversamente proporcional al cuadrado de la distancia. Esto se produce en una dirección determinada en la que emite una fuente luminosa (Álvarez, 2015, pág. 12). Al interpretar esta definición se deduce que la fuente emisora de luz produce una iluminación que a medida que se aleja disminuye, pero lo intensidad luminosa (I) permanece constante. Por ejemplo, si tenemos una intensidad de luminosa de $36 \mathrm{~cd}$, para superficies situadas a 1, 2 y 3 metros de distancia, la iluminación sería 36 Lux, 9 Lux y 4 Lux, respectivamente. Esto se debe a la iluminación es inversamente al cuadrado de la distancia a las superficies en las que incide la luz.

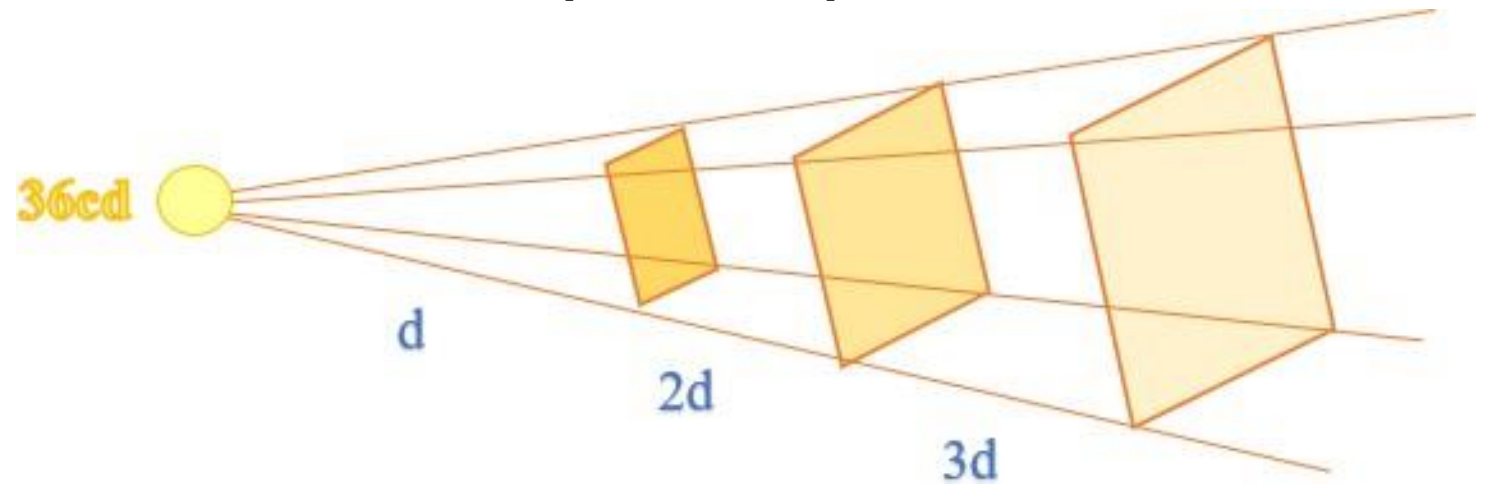

Figura 10. Ubicación de superficies

La iluminación de una superficie a medida que se aleja de su zona de incidencia disminuye considerablemente. Por el método de punto por punto se determina la iluminación o luminancia desde la recta normal a la superficie variando los ángulos de separación.
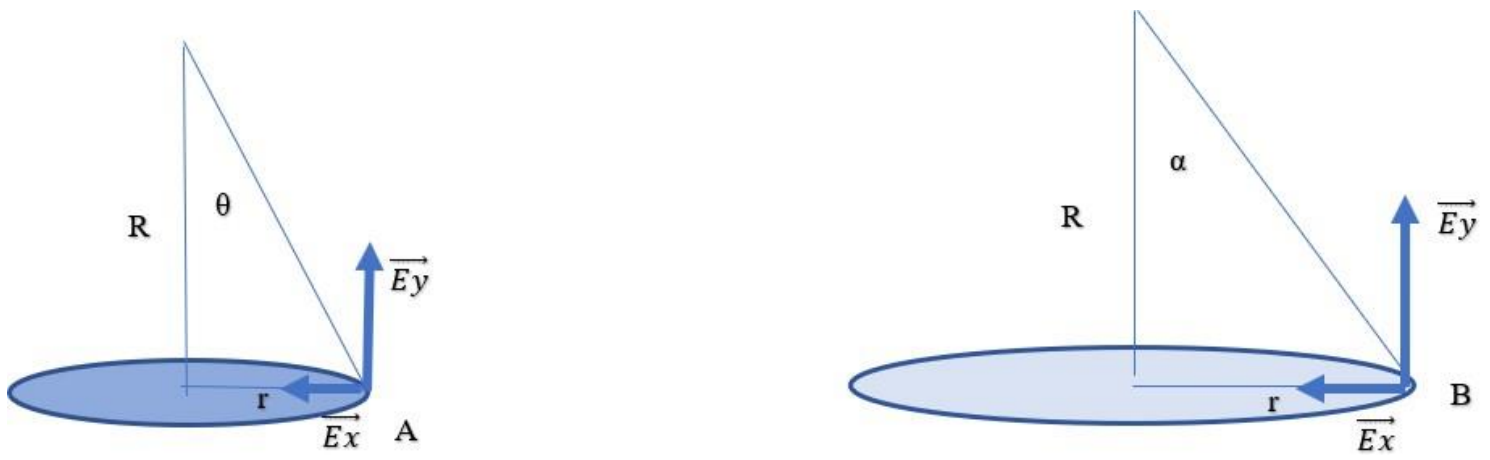

Figura 11. Luminosidad de superficies en diferentes puntos

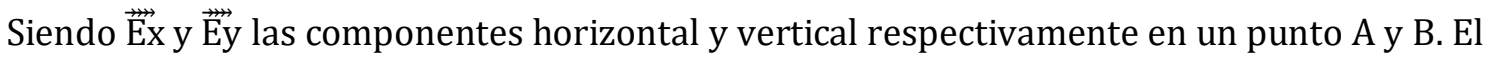
ángulo respecto a la normal a la superficie varía a medida que se aleja en las posiciones $\mathrm{A}$ y $B$ respectivamente, luego se cumple que: $\theta>\alpha$.

\section{Método punto por punto}

Para lograr un cambio cognitivo en el estudio de la luminancia se utiliza el método punto por punto que analiza un punto en cualquier parte del área de incidencia de la luz. Para el efecto se hace una evocación de lo que se sabe y no se sabe detalladamente acerca de las características favorables y no favorables de dos bombillas de diferente naturaleza. Para que el aprendizaje obtenido sea a largo plazo se basa en la experimentación en el Centro de Física de la Universidad Central del Ecuador. Los conocimientos previos del tema además de la constatación de los datos para el entendimiento de la iluminación producida por una bombilla. En primer lugar, se mide la altura del techo en el que se suspende la luminaria que 
está a $3 \mathrm{~m}$ aproximadamente, luego se considera la observación de una bombilla de incandescencia de $100 \mathrm{~W}$ (vatios) y otra fluorescente de $40 \mathrm{~W}$ (vatios), siendo respectivamente la Intensidad luminosa de $130 \mathrm{~cd}$ y $200 \mathrm{~cd}$ respectivamente.

Los centros docentes tienen unos requisitos específicos de iluminación, entre otras cosas por el tipo de actividades que en ellos se realizan. Una deficiente iluminación de las instalaciones de un centro docente, y en especial de las aulas y espacios destinados a impartir clases, aprendizaje y estudio, puede ocasionar fatiga visual, lesiones en la vista e incluso podría ser causa del incremento del índice de fracaso escolar por bajo rendimiento de los alumnos.

\begin{tabular}{lc}
\hline \multicolumn{2}{l}{ Cuadro luminancia en lux en un centro docente } \\
\multicolumn{1}{c}{ Sitio } & $\underline{\text { Luminancia }}$ \\
& 250 a 1000 lux \\
Laboratorio & 350 a 1000 lux \\
Alumbrado general en aulas & 300 a 700 lux \\
Pizarras & 200 y 1000 lux \\
Salas de Conferencias & 300 y 750 lux \\
Bibliotecas
\end{tabular}

Cuadro 1. Niveles recomendados de iluminación por zonas. Adaptado de (Helios Strategia Ecuador)

Por lo anteriormente expuesto se deduce que lo óptimo en nuestra experiencia seria de aproximadamente 400 Lux.

\subsection{Primer paso}

Para el caso en que no es conocido el ángulo del rayo luminoso con la vertical se calcula de la siguiente manera:

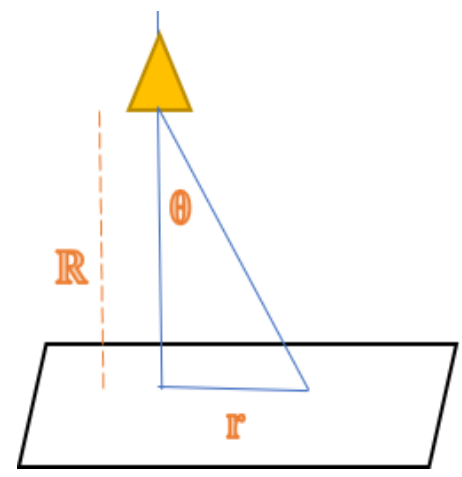

Figura 12. Ángulo respecto a la vertical

$$
\tan \theta=\begin{aligned}
& \mathrm{r} \\
& \overline{\mathrm{R}}
\end{aligned}
$$

Ecuación 10

\subsection{Segundo paso}

En este paso se determina la I (intensidad de flujo luminoso según la dirección del punto a la fuente). Para ello, se tiene que tener elegida tanto el tipo de lámpara como el tipo de luminaria. Una vez con estos datos, se consulta con el fabricante de luminarias la curva fotométrica o curva de distribución luminosa. Generalmente, dicha información se puede consultar en cualquier catálogo online de fabricantes de luminarias técnicas. 


\begin{tabular}{lccc}
\hline Tipo de lámpara & Potencia & $\frac{\text { Intensidad }}{\text { luminosa }}$ & $\frac{\text { Identificación }}{\text { gráfica }}$ \\
\hline $\begin{array}{l}\text { Lámparas } \\
\text { incandescentes }\end{array}$ & $100 \mathrm{~W}$ & $130 \mathrm{~cd}$ & \\
$\begin{array}{l}\text { Lamparas } \\
\text { fluorescentes }\end{array}$ & $40 \mathrm{~W}$ & $200 \mathrm{~cd}$ & \\
\hline
\end{tabular}

Cuadro 2. Flujo luminoso en candelas. Cuadro comparativo. Adaptado de (Tablas y comparativos, s.f.)

Se elige una luminaria que contiene una lámpara de $100 \mathrm{~W}$, su eficiencia luminosa es de $58 \mathrm{~lm} / \mathrm{W}$ y con una intensidad luminosa de $130 \mathrm{~cd}$. Así mismo se eligen una bombilla fluorescente de $40 \mathrm{~W}$ y una intensidad luminosa de $200 \mathrm{~cd}$.; por lo tanto, las dos lámparas compactas que en total dan un flujo luminoso para las dos bombillas.

$$
\begin{gathered}
\mathrm{F}=100 \mathrm{~W} \times \frac{58 \mathrm{~lm}}{\mathrm{~W}}=5800 \mathrm{~lm} \\
\text { Ecuación } 11 \\
\mathrm{~F}=40 \mathrm{~W} \times \frac{58 \mathrm{~lm}}{\mathrm{~W}}=2320 \mathrm{~lm} \\
\text { Ecuación } 12
\end{gathered}
$$

Lo que demuestra que el flujo luminoso es mayor en el foco de incandescencia. Cabe indicar que la intensidad luminosa se establece por medio de una unidad patrón, como se mencionó en el patrón luminoso producido por el metal platino a $1773^{\circ} \mathrm{C}$. Con base en este dato se verifica que los fabricantes de bombillas eléctricas indican la intensidad luminosa en candelas "cd". En el caso de prescindir de este dato, se procede a verificar si el fabricante indica el flujo luminoso en lumen " $\mathrm{lm}$ " y se tiene que establecer el ángulo sólido en estereorradián "sr".

\subsection{Tercer paso}

En el método punto por punto se mide un ángulo respecto a la normal de la superficie y se calcula la iluminación horizontal y vertical con las siguientes expresiones:

$$
\mathrm{Ex}=\frac{\mathrm{I} \cos ^{3} \emptyset}{\mathrm{R}^{2}} \quad[\mathrm{Lux}] ; \quad \mathrm{Ey}=\frac{\mathrm{I} \cos ^{2} \emptyset \operatorname{sen} \emptyset}{\mathrm{R}^{2}} \quad[\mathrm{Lux}]
$$

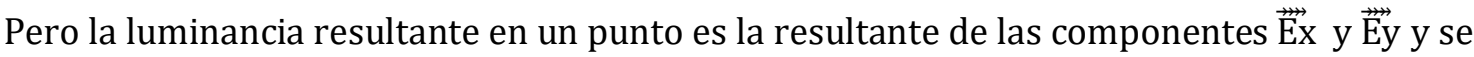
la obtiene aplicando el teorema de Pitágoras:

$$
\begin{aligned}
\vec{E}= & \sqrt{\vec{E} \mathrm{x}^{2}+\vec{E} \mathrm{y}^{2}} \\
& \text { Ecuación } 14
\end{aligned}
$$

\subsection{Cuarto paso (resultados obtenidos)}

Aplicamos las ecuaciones anteriores a la experiencia de medición de la iluminancia de una bombilla de incandescencia y la bombilla fluorescente. Las medidas de una lámpara de incandescencia de 100 vatios posee una intensidad luminosa de $130 \mathrm{~cd}$ y la fluorescente de 
40 vatios de intensidad luminosa de $200 \mathrm{~cd}$. El estudiante establece que en un salón clases el techo está a $3 \mathrm{~m}$, su intención es saber la luminosidad en una superficie, por lo tanto, el estudiante establece los siguientes parámetros de medida a diferentes ángulos de separación respecto a la línea normal a la superficie:

\begin{tabular}{cccc}
\hline \multicolumn{4}{c}{ FOCO DE INCANDESCENCIA DE 130 cd } \\
\hline$\Theta$ (grados) & Ex (Lux) & Ey (Lux) & E total (Lux) \\
$0^{\circ}$ & 14,40 & 0 & 14,4 \\
$30^{\circ}$ & 9,38 & 5,41 & 10,82 \\
$45^{\circ}$ & 5,10 & 5,10 & 7,21 \\
$60^{\circ}$ & 1,80 & 3,12 & 3,60 \\
$75^{\circ}$ & 0,25 & 0,93 & 0,96 \\
$90^{\circ}$ & 0 & 0 & 0 \\
& FOCO FLUORESCENTE DE 200 cd & \\
$\Theta($ grados) & Ex (Lux) & Ey (Lux) & E total (Lux) \\
& & 0 & 22,22 \\
$0^{\circ}$ & 22,22 & 8,33 & 16,66 \\
$30^{\circ}$ & 14,43 & 7,85 & 7,85 \\
$45^{\circ}$ & 7,85 & 4,81 & 5,55 \\
$60^{\circ}$ & 2,77 & 1,43 & 2,18 \\
$75^{\circ}$ & 0,38 & 0 & 0 \\
$90^{\circ}$ & 0 &
\end{tabular}

Cuadro 3. Cálculo de datos

El diagrama "Isolux" indica la incidencia de luz en una superficie a partir de la luminosidad del centro hacia el exterior del círculo que ilumina la lámpara:

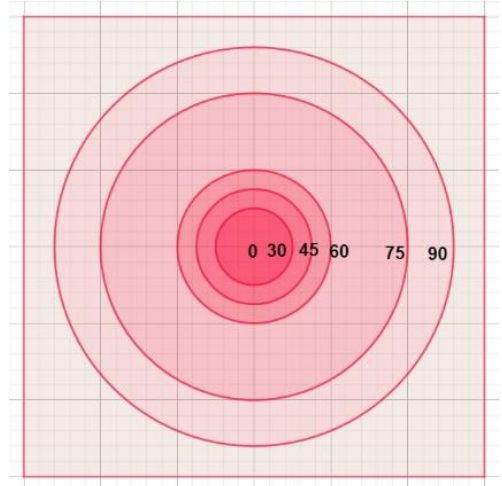

Figura 13. Diagrama Isolux de iluminación superficial

\section{Conclusiones}

El Flujo Luminoso es menor en la bombilla fluorescente, mientras que en la bombilla de incandescencia es mayor, a diferencia de la luminancia que es mayor para la bombilla fluorescente. Esto indica que el flujo luminoso es inversamente proporcional a la luminancia comparada de las dos bombillas. 
La bombilla fluorescente produce mayor luminancia en los puntos estudiados, por lo tanto, es mucho más eficiente además de ser mucho más económico por su bajo consumo de aproximadamente $1 / 6$ del consumo en vatios de la bombilla de incandescencia. Los cálculos pueden variar por diversos factores, pues hay lugares en los que la altura de la habitación que se tomó como muestra en ciertas partes se media altura de 2,94 m y otras de 2,98 m, pero para términos de cálculos se estableció la medida que indicara los ayudantes del Centro de Física de la Universidad Central. También la luminancia varía por la absorción de radiación de la superficie incidente o la rugosidad de la misma.

En el punto de ángulo $0^{\circ}$ respecto a la vertical se produce la mayor iluminación horizontal de 14.4 Lux a 3 metros de altura, mientras que la bombilla fluorescente a la misma altura de tres metros y $0^{\circ}$ presenta una luminancia de 22,22 Lux. Por lo que la eficiencia en luminancia en los dos casos estudiados en el laboratorio se debe colocar al menos 18 bombillas fluorescentes o 28 bombillas incandescentes.:

18 bombillas fluorescentes x 22,22 Lux $=399,96$ Lux.

28 bombillas incandescentes $\mathrm{x} 14,44 \mathrm{Lux}=403,2 \mathrm{Lux}$

Estos datos son hipotéticos y partimos del supuesto que no existe superposición de ondas entre las bombillas constructiva y/o destructiva. Esto demuestra la mayor eficacia y eficiencia de las bombillas fluorescentes, ya que garantizan ahorro de consumo eléctrico, mayor intensidad luminosa y la utilización 10 bombillas menos. Además, el fabricante garantiza mayor número de horas de duración por lo que es altamente recomendable.

Las bombillas fluorescentes son de luz blanca o luz cálida, y sabiendo que el ojo humano es más sensible a la luz amarilla de $555 \mathrm{~nm}$. Esto indica que es más recomendable el uso de bombillas de luz cálida, pero puede ir a gusto de la persona que realizan sus actividades en ese lugar de estudio.

A la hora de aplicar el método de punto por punto se concluye que su utilización se limita a conocer la iluminancia en puntos concretos basados en los conocimientos previos estudiados en clase y por experiencia en el manejo de bombillas de uso doméstico. Luego de ser un sujeto activo en la experimentación y medición adquiere una nueva información observando que una superficie está conformada por miles de puntos por lo que las respuestas son variadas a diferentes ubicaciones, más aún si se utilizan bombillas de diferente tipo. El aprendizaje significativo en el estudio de la luz e iluminación se da cuando la teoría va ligada estrechamente de la práctica, la obtención de nueva información y la capacidad de evocar sus conocimientos y así lograr un feedback o retroalimentación sucesiva. 


\section{Bibliografía}

Alvarez, T. (2015). Iluminación en el puesto de trabajo. Criterios para la evaluación y acondicionamiento de los puestos. Madrid: INSHT.

Ausubel, D. (1963). Psicología Educativa. Un punto de vista cognoscitivo. México:Trillas segunda edición

Borja Reyes, A. G. (2017). Confort lumínico en los espacios interiores de la biblioteca de la ciudad y provincia, en la ciudad de Ambato. Ambato.

Dominguez Martínez, F. (2008). Instalaciones eléctricas de alumbrado e industriales. España: Paraninfo.

INSHT, I. N., \& Alvarez Bayona, T. (2015). Iluminación en el puesto de trabajo. Madrid: NIPO.

León, A. (2002). “Manual de luminotecnia”. España: Edición España.

Ley orgánica de Educación Superior (LOES). (2018). Recuperado el 23 de agosto de 2019 de http://aka-cdn.uce.edu.ec/ares/tmp/Elecciones/2\%20LOES.pdf

Ministerio de Educación del Ecuador. (2008). Ley Orgánica de Educación Superior (LOES). Quito, Ecuador.

Rodríguez Palmer, M. L. (2004). La Teoría del Aprendizaje Significativo. Obtenido de http://cmc.ihmc.us/papers/cmc2004-290.pdf

Tablas y comparativos. (s.f.). Obtenido de Apuntes Científicos: http://apuntescientificos.org/tablas-iluminacion.html

Tippens, P. (2007). Física, Conceptos y Aplicaciones. Séptima edición. México: McGRAWHILL/INTERAMERICANA EDITORES, S.A. DE C.V.

Young, H. D., \& Roger A, F. ((2009)). Física universitaria, con física moderna volumen 2. Decimosegunda edición. México: PEARSON EDUCACIÓN. 


\section{Autores}

JOSÉ RICARDO AULESTIA-ORTIZ obtuvo su título de Magister en Instituciones de Educación Superior en la Escuela Politécnica Nacional. Ingeniero en Gestión Educativa en la Universidad Metropolitana de Quito. Licenciado en Ciencias de la Educación especialidad Matemáticas y Física. Profesor de Física en la carrera de Pedagogía de las Ciencias Experimentales, Matemática y Física.

Actualmente es profesor en la Universidad Central del Ecuador, fue profesor de Física Medica en la Universidad Tecnológica Equinoccial. Ha sido Rector de la Academia Aeronáutica Elia Liut y del Colegio Agropecuario Genoveva German. Ha trabajado en el sector público como Director de Educación en el GAD del Cantón Mejía. Fue Profesor de Física del Colegio Militar Eloy Alfaro y en el Instituto Superior Nelson Torres.

NELSON MEJÍA-TORRES es egresado de la Carrera de Matemática y Física, se encuentra en proceso de titulación en la Carrera de Matemática y Física.

Realizó sus pasantías en la cátedra de Electromagnetismo y Física Moderna.

SHIRLEY VERA-MACÍAS es egresada de la Carrera de Matemática y Física, se encuentra en proceso de titulación en la Carrera de Matemática y Física.

Realizó sus pasantías en la cátedra de Electromagnetismo y Física Moderna y colaboró en el Centro de Física.

LUIS PUGA-PEÑA. Obtuvo su título de Licenciado en Ciencias de la Educación Especialidad Matemáticas y Física, en la Universidad Central del Ecuador; y su título de Magister en Docencia Matemática, en la Universidad Técnica de Ambato.

Actualmente es docente de la Universidad UTE Ecuador y de la Universidad Central del Ecuador en la Facultad de Ciencias Económicas. Autor y coautor de artículos científicos sobre educación. Escritor del Libro Ecuaciones Algebraicas 